\title{
Wolbachia co-infection in a hybrid zone: Discovery of horizontal gene transfers from two Wolbachia supergroups into a grasshopper genome
}

Lisa J Funkhouser-Jones, Stephanie R Sehnert, Paloma Martínez-Rodríguez, Raquel Toribio-Fernández, Miguel Pita, José L Bella, Seth R Bordenstein

Hybrid zones and the consequences of hybridization have contributed greatly to our understanding of evolutionary processes. Hybrid zones also provide valuable insight into the dynamics of symbiosis since each subspecies or species brings its unique microbial symbionts, including germline bacteria such as Wolbachia, to the hybrid zone. Here, we investigate a natural hybrid zone of two subspecies of the meadow grasshopper Chorthippus parallelus in the Pyrenees Mountains. We set out to test whether co-infections of $B$ and $F$ Wolbachia in hybrid grasshoppers enabled horizontal transfer of phage WO, similar to the numerous examples of phage WO transfer between A and B Wolbachia coinfections. While we found no evidence for transfer between the divergent co-infections, we discovered horizontal transfer of at least three phage WO haplotypes to the grasshopper genome. Subsequent genome sequencing of uninfected grasshoppers uncovered the first evidence for two discrete Wolbachia supergroups ( $B$ and $F$ ) contributing at least $448 \mathrm{~kb}$ and $144 \mathrm{~kb}$ of DNA, respectively, into the host nuclear genome. Fluorescent in situ hybridization verified the presence of Wolbachia DNA in C. parallelus chromosomes and revealed that some inserts are subspecies-specific while others are present in both subspecies. We discuss our findings in light of symbiont dynamics in an animal hybrid zone. 
1 Wolbachia Co-infection in a Hybrid Zone: Discovery of Horizontal Gene Transfers from Two

2 Wolbachia Supergroups into an Animal Genome

3

4 Running Title: Wolbachia HGT in a Grasshopper Genome 5

6 Lisa J. Funkhouser-Jones ${ }^{1, *}$, Stephanie R. Sehnert ${ }^{1}$, Paloma Martínez-Rodríguez ${ }^{3,4}$, Raquel 7 Toribio-Fernández ${ }^{3}$, Miguel Pita ${ }^{3}$, José L. Bella ${ }^{3}$, and Seth R. Bordenstein ${ }^{1,2, *}$

9 Affiliations:

$10{ }^{1}$ Department of Biological Sciences, Vanderbilt University, Nashville, Tennessee, USA

11 2Department of Pathology, Microbiology and Immunology, Vanderbilt University, Nashville, 12 Tennessee, USA

13 32Departamento de Biología (Genética), Facultad de Ciencias, Universidad Autónoma de Madrid, 14 Madrid, Spain

15 Current address: INRA, Univ. Nice Sophia Antipolis, CNRS, UMR 1355-7254 Institut Sophia 16 Agrobiotech, Sophia Antipolis, France 17

$18{ }^{*}$ Correspondence and requests for materials should be addressed to:

19 Seth Bordenstein | s.bordenstein@vanderbilt.edu | 615-322-9087

20 Lisa Funkhouser-Jones | lisa.j.funkhouser@vanderbilt.edu | 615-322-9094 


\section{ABSTRACT}

22 Hybrid zones and the consequences of hybridization have contributed greatly to our

23 understanding of evolutionary processes. Hybrid zones also provide valuable insight into the

24 dynamics of symbiosis since each subspecies or species brings its unique microbial symbionts,

25 including germline bacteria such as Wolbachia, to the hybrid zone. Here, we investigate a natural

26 hybrid zone of two subspecies of the meadow grasshopper Chorthippus parallelus in the

27 Pyrenees Mountains. We set out to test whether co-infections of B and F Wolbachia in hybrid

28 grasshoppers enabled horizontal transfer of phage WO, similar to the numerous examples of

29 phage WO transfer between A and B Wolbachia co-infections. While we found no evidence for

30 transfer between the divergent co-infections, we discovered horizontal transfer of at least three

31 phage WO haplotypes to the grasshopper genome. Subsequent genome sequencing of uninfected

32 grasshoppers uncovered the first evidence for two discrete Wolbachia supergroups (B and F)

33 contributing at least $448 \mathrm{~kb}$ and $144 \mathrm{~kb}$ of DNA, respectively, into the host nuclear genome.

34 Fluorescent in situ hybridization verified the presence of Wolbachia DNA in C. parallelus

35 chromosomes and revealed that some inserts are subspecies-specific while others are present in

36 both subspecies. We discuss our findings in light of symbiont dynamics in an animal hybrid

37 zone. 


\section{INTRODUCTION}

Microbial communities of many arthropod species are dominated numerically by heritable bacterial symbionts whose phenotypic effects range from mutualism to parasitism (Douglas 2011). In some cases, millennia of co-evolution have produced obligate, mutualistic relationships in which microbial symbionts make essential amino acids and/or vitamins to complement the nutritionally incomplete diet of their hosts (Pais et al. 2008; Tamas et al. 2002; van Ham et al. 2003). In other cases, maternally-transmitted bacteria directly impact arthropod host reproduction by manipulating sex determination, fecundity, and the ratio of infected females (the transmitting-sex) within a population (LePage \& Bordenstein 2013). The alphaproteobacterium Wolbachia is the most widespread of these reproductive manipulators, infecting an estimated $40-52 \%$ of all terrestrial arthropod species (Weinert et al. 2015; Zug \& Hammerstein 2012). It uses a variety of mechanisms to increase the number of host females in a population including feminization of genetic males, male-killing, parthenogenesis, and cytoplasmic incompatibility (CI), which typically results in embryonic death of offspring produced by an uninfected female mated with an infected male (Serbus et al. 2008).

Hybrid zones are excellent model systems for studying the impact of interactions between heritable endosymbionts on animal evolution. For example, Drosophila recens and $D$. subquinaria meet in secondary contact in a hybrid zone spanning central Canada where $D$. recens is infected by a Wolbachia strain that causes strong CI ( $\sim 90 \%$ reduction in progeny) when males mate with naturally uninfected D. subquinaria females (Jaenike et al. 2006; Shoemaker et al. 1999). In contrast, weak levels of CI in a hybrid zone could promote Wolbachia exchange between animal species. Two closely related species of field crickets, Gryllus firmus and G. pennsylvanicus, hybridize in a north-south zone along the eastern front of the Appalachian Mountains in the United States (Harrison \& Arnold 1982). Though each cricket species is predominantly infected with different Wolbachia strains, Wolbachia is not a primary source of hybrid incompatibility in this system (Mandel et al. 2001). This may partly explain why a significant portion of G. pennsylvanicus are infected with both Wolbachia strains (Mandel et al. 2001). Wolbachia co-infection of the same host can readily facilitate gene exchange and transfer of mobile elements between intracellular bacteria according to the intracellular arena concept (Bordenstein \& Reznikoff 2005; Bordenstein \& Wernegreen 2004; Newton \& Bordenstein 2011). Indeed, we previously showed that the co-infecting Wolbachia strains in $G$. 
69

70

pennsylvanicus crickets harbor a nearly identical infection of Wolbachia's temperate bacteriophage WO (Chafee et al. 2010). Thus, hybrid zones that permit mixing of Wolbachia symbionts may in turn enable horizontal gene transfer between the coinfections.

Here, we investigate horizontal gene transfer of bacteriophage WO in a natural hybrid zone of the meadow grasshopper Chorthippus parallelus. During the last Ice Age, C. parallelus populations on the Iberian Peninsula were geographically isolated from those in continental Europe, resulting in the divergence of Iberian C. parallelus erythropus (Cpe) subspecies from the contemporary continental subspecies, C. parallelus parallelus (Cpp) (Shuker et al. 2005a). Now in secondary contact, hybrids of the two subspecies have interbred for an estimated 9,000 generations along a hybrid zone in the Pyrenees Mountains between France and Spain (Hewitt 1993; Shuker et al. 2005a) (Figure 1). Due to low dispersal rates, all grasshoppers collected from populations in the hybrid zone (i.e., Portalet) are hybrids of the two subspecies, while pure Cpp and Cpe populations reside on the edges of the hybrid zone (Gabas for Cpp and Escarrilla for Cpe) (Bella et al. 2007; Hewitt 1993; Shuker et al. 2005a) (Figure 1). F1 hybrids produced in laboratory crosses between the subspecies follow Haldane's rule and produce sterile F1 hybrid males, but both hybrid males and females in the field are fertile, possibly due to selection against deleterious allelic combinations that result in hybrid sterility (Bella et al. 1990; Shuker et al. 2005b).

C. parallelus subspecies are infected with Wolbachia strains from two divergent supergroups: Cpp are primarily infected with B Wolbachia while Cpe mostly harbor F Wolbachia (Zabal-Aguirre et al. 2010). In natural hybrid populations, the B and F Wolbachia each cause a significant amount of unidirectional CI, reducing embryo viability by approximately 33\% and 23\%, respectively, in incompatible crosses (Zabal-Aguirre et al. 2014). Bidirectional CI is weaker, with a $15 \%$ reduction in viable embryos in crosses between Finfected and B-infected grasshoppers (Zabal-Aguirre et al. 2014). With these incomplete CI rates permitting the mixture of Wolbachia strains, the incidence of Wolbachia infection is highly variable in the hybrid zone, and individuals collected from a single population are either uninfected, singly-infected with B or F Wolbachia, or co-infected by both (Zabal-Aguirre et al. 2010). supergroup co-infections in arthropods (Bordenstein \& Wernegreen 2004; Chafee et al. 2010; 
100 Gavotte et al. 2007; Kent et al. 2011; Masui et al. 2000; Metcalf \& Bordenstein 2012), we used

101 the C. parallelus hybrid zone to investigate whether phage WO can also transfer between co-

102 infections of B and F Wolbachia. Here, we present the first screen for phage WO in the $C$.

103 parallelus hybrid zone. While we do not find evidence for WO transfer between B and F

104 Wolbachia, we identify three main WO haplotypes in the grasshopper genome. We also report,

105 for the first time to our knowledge, the transfer of large amounts of DNA from two divergent

106 Wolbachia supergroups into the host nuclear genome. 
107 MATERIALS AND METHODS

108 Sample collection, DNA extraction, and Wolbachia strain typing

109 The Spanish Comunidad de Madrid, the Gobierno de Aragón and the French Parc

110 National des Pyrénées gave permission (permit numbers 10/103410.9/15; INAGA

111 500201/24/2012/12140; and Autorisation 2015-9, respectively) to collect Chorthippus parallelus

112 individuals from five European and Iberian populations (Figure 1). Gonads (or the whole body)

113 were dissected and fixed in 100\% ethanol. DNA was extracted as described elsewhere (Martinez-

114 Rodriguez et al. 2013). Wolbachia was detected by PCR amplification of the Wolbachia 16S

115 rRNA gene using Wolbachia-specific primers (Zabal-Aguirre et al. 2010), followed by nested

116 PCR amplifications using B and F supergroup-specific primers (Martinez-Rodriguez et al. 2013).

$11710 \mu \mathrm{l}$ of each amplification product were electrophoretically separated on $1 \%$ agarose gels,

118 which were stained with $0.5 \mathrm{mg} / \mathrm{ml}$ ethidium bromide and visualized under UV light (UVIdoc,

119 Uvitec Cambridge).

120

121 Phage PCR amplification, cloning and sequencing

$122 \quad$ All PCR amplifications for phage and Wolbachia gene analyses were performed using

$1237.5 \mu \mathrm{l}$ 2X GoTaq Green Master Mix (Promega), $3.6 \mu 1$ sterile water, $1.2 \mu 1$ of each primer $(5 \mu \mathrm{M})$

124 and $1.5 \mu \mathrm{l}$ template DNA for a $15 \mu \mathrm{l}$ total reaction volume (scaled up as necessary) on a Veriti

125 Thermal Cycler (Applied Biosystems) with the following primers: phgWOF (5'-

126 CCCACATGAGCCAATGACGTCTG-3') and phgWOR (5'-

127 CGTTCGCTCTGCAAGTAACTCCATTAAAAC-3') for the WO minor capsid gene (Masui et

128 al. 2001); WolbF (5'-GAAGATAATGACGGTACTCAC-3') and WolbR3 (5'-

129 GTCACTGATCCCACTTTAAATAAC-3') for the Wolbachia 16S ribosomal RNA gene

130 (Casiraghi et al. 2001); ftsZunif (5'-GGYAARGGTGCRGCAGAAGA-3') and ftsZunir (5'-

131 ATCRATRCCAGTTGCAAG-3') for Wolbachia ftsZ (Lo et al. 2002). The following primers

132 were designed as part of this study to amplify specific WO alleles: forward primer WOPar1_F1

133 (5'-AATCTAAAAAGCGAAGTGAATCGTT-3') paired with phgWOR to amplify Cpar-WO1

134 alleles; reverse primer WOPar3_R1 (5'-CGACAGTTCTCGTAGCCTTCCTCA-3') paired with

135 phgWOF to amplify Cpar-WO3 alleles.

136 To clone and sequence the orf7 gene, PCR products were run on a 1\% TBE agarose gel,

137 then excised and purified using the Wizard PCR and Gel Clean-up Kit (Promega). $4 \mu$ of each 
138 purified PCR product was cloned into a pCR4-TOPO vector using the TOPO TA Cloning kit 139 (Invitrogen). OneShot TOP10 E. coli cells (Life Technologies) were transformed with the

140 recombinant plasmids through heat shock according to the manufacturer's protocol. Transformed

141 E. coli were plated on LB + carbenicillin plates and incubated overnight at $37^{\circ} \mathrm{C}$. Fifteen to 26

142 colonies were picked per plate then sent to GENEWIZ, Inc. (South Plainfield, NJ) for plasmid

143 purification and Sanger sequencing. Both forward and reverse directions were sequenced for

144 each plasmid then assembled in Geneious v5.5.8. For Sanger sequencing with allele-specific

145 primers, PCR products were excised and purified from agarose gels as described above then sent

146 to GENEWIZ, Inc. for sequencing. Both forward and reverse directions were sequenced for each

147 PCR product then assembled in Geneious v5.5.8.

Phylogenetic tree construction

All multiple sequence alignments and phylogenetic trees were constructed in Geneious v5.5.8. Minor capsid sequences obtained through cloning and/or Sanger sequencing were aligned with homologous sequences from other WO phages (Table S3) using the Translation Align Tool with default parameters, and the $d n a A$ and $f a b G$ contigs from high-throughput sequencing were aligned with their homologs in Wolbachia strains using the Geneious alignment tool with default parameters. Wolbachia $d n a A$ and $f a b G$ genes were extracted from full genome sequences from NCBI (Genbank) as follows: $w \mathrm{Ha}$ [CP003884.1], $w \mathrm{Mel}$ [AE017196.1], $w \mathrm{Ri}$ [CP001391.1], wNo [CP003883.1], $w$ Pip strain Pel [AM999887.1], wOo [HE660029.1], wOv strain Cameroon [HG810405.1], wBm strain TRS [AE017321.1], and $w$ Cle [AP013028.1].

After indels were manually removed, the minor capsid gene alignment was $332 \mathrm{bp}$ with 49 sequences, the $d n a A$ alignment was 742 bp with 11 sequences, and the fabG alignment was 735 bp with 11 sequences. 'N"'s were added to the 5' or 3' ends of any sequences that were shorter than the total alignment length. jModelTest 0.1.1 was used to determine the best model of nucleotide evolution for each alignment based on the corrected Akaike information criterion (AICc). For each gene, PhyML (Guindon \& Gascuel 2003) and MrBayes (Huelsenbeck \& Ronquist 2001) were executed in Geneious with default parameters to construct a maximum likelihood tree with bootstrapping and a Bayesian tree with a burn-in of 100,000, respectively.

167 For the minor capsid gene, the third best model of nucleotide evolution $(\mathrm{HKY}+\mathrm{G})$ was used to generate both the maximum likelihood and Bayesian trees since the first two best models were 
169 not available in PhyML or MrBayes. The Hasegawa-Kishino-Yano (HKY) model of nucleotide

170 evolution allows variable base frequencies and separate rates for transitions and transversions

171 (Hasegawa et al. 1985). For the $d n a A$ gene, the $10^{\text {th }}$ best model of HKY $+\mathrm{G}$ was used since the

172 first 9 were not available in PhyML or MrBayes. For the $f a b G$ gene, the second best model of

173 GTR + G was used. The general time reversible (GTR) model of nucleotide evolution allows

174 variable base frequencies and assumes a symmetric substitution matrix (Lanave et al. 1984;

175 Tavare 1986). For both the HKY and GTR models, rate variation among sites was modeled as a

176 gamma distribution $(+\mathrm{G})$.

177

178 High throughput sequencing of Wolbachia genomic inserts

179 Pooled DNA from three uninfected grasshoppers (two gonadal and one whole-body

180 extractions) from the Gabas population (pure Cpp) was sequenced as $100 \mathrm{bp}$, paired-end reads on

181 a single lane of an Illumina HiSeq2000 at the Vanderbilt VANTAGE sequencing facility. All

182 analysis of sequencing data was performed in CLC Genomics Workbench 8. Reads were

183 trimmed based on a quality limit of 0.05 and minimum length of $50 \mathrm{bp}$. After trimming, the data

184 consisted of 227,349,258 reads with an average length of $93.5 \mathrm{bp}$ totaling 21,347,095,705 bp.

185 All reads were initially mapped to the B Wolbachia genome of $w$ Pip strain Pel (Genbank

186 AM999887) using the CLC mapping tool with the following parameters: 80\% similarity over

$18780 \%$ read length, mismatch $\cos t=2$, insertion $\cos t=3$, deletion $\cos t=3$, and random mapping

188 of non-specific reads. To ensure that the mapped reads were indeed from Wolbachia, reads from

189 core Wolbachia genes were searched against the NCBI nucleotide database using blastn

190 (megablast). Since many of the reads were more similar to genomic sequences from the $\mathrm{F}$

191 Wolbachia $w$ Cle than to $w$ Pip or other B Wolbachia genomes, we re-mapped all reads to the

192 wCle (Genbank AP013028) and wPip (Genbank AM999887) reference genomes simultaneously

193 with more stringent parameters: $90 \%$ similarity over $90 \%$ read length, mismatch cost $=2$,

194 insertion cost $=3$, deletion cost $=3$, and random mapping of non-specific reads. Since read

195 mapping to each genome was mutually exclusive, this generated a list of reads that preferentially

196 mapped to one genome over the other. To ensure that this was the case, reads that mapped to

$197 w$ Pip were extracted and mapped to the $w$ Cle genome and vice versa with the more stringent

198 parameters (90\% similarity over 90\% read length) to generate a combined list of "non-specific

199 reads". After excluding these non-specific reads, the remaining reads were mapped back to the 
200

201

202

203

204

205

206

207

208

209

210

211

212

213

214

215

216

217

218

219

220

221

222

223

224

225

226

227

228

229

230

genome that they preferentially mapped to in order to determine the final lengths of the $\mathrm{B}$ and $\mathrm{F}$ inserts.

To find genes shared between the inserts, we took the reads that preferentially mapped to either $w$ Pip or $w \mathrm{Cle}$ (B and $\mathrm{F}$ reads, respectively) and mapped them with less stringent parameters ( $70 \%$ sequence similarity over $90 \%$ sequence length) to the reciprocal genome.

Genes were considered shared between the two inserts if both $\mathrm{B}$ and $\mathrm{F}$ reads mapped to homologous genes on both the $w$ Pip and $w$ Cle genomes and total read length for both $\mathrm{B}$ and $\mathrm{F}$ reads on each gene exceeded $80 \mathrm{bp}$. B and $\mathrm{F}$ variants for each gene were manually verified by using blastn (discontiguous megablast) to confirm that percent similarity of B variants to $w$ Pip were higher than to $w \mathrm{Cle}$ and vice versa.

To determine whether reads preferentially mapped to $w$ Pip and $w$ Cle over Wolbachia strains from other supergroups, we mapped all reads simultaneously to $w \mathrm{Pip}, w \mathrm{Cle}, w \mathrm{Mel}, w \mathrm{Bm}$, and $w$ Oo reference genomes with a cutoff of $90 \%$ sequence similarity over $90 \%$ read length or $65 \%$ similarity over $80 \%$ read length. All reads that ambiguously mapped to more than one location were discarded.

Visualization of read mapping coverage on the $w$ Pip and $w$ Cle circular genomes was generated using the BLAST Ring Image Generator v0.95 (Alikhan et al. 2011) with a maximum mapping coverage of 30 .

\section{FISH analysis}

To perform the cytogenetic analyses, male adult specimens of Cpp and Cpe were collected from the Gabas (France) and Escarrilla (Spain) populations, respectively. Grasshopper gonads were extracted and fixed in fresh ethanol:acetic acid (3:1) and used to prepare slides. After identifying uninfected individuals with Wolbachia-specific primers, as mentioned above, we designed primers to amplify a Wolbachia contig (Cpar-Wb1) identified during genome sequencing (Table S6): 177contigF (5'-ACAGGAATTACAGCCTCAGGT-3') and 177contigR (5'-AAAAGCGTGGCAACAAAGTT-3'). PCR amplifications used the following conditions: Buffer 1X, $\mathrm{MgCl}_{2} 2 \mathrm{mM}$, dNTPs (Roche) $0.2 \mathrm{mM}, 1.2 \mu \mathrm{M}$ of each primer, BIOTAQ DNA polymerase $1.25 \mathrm{U}$ (Biotools), and $100 \mathrm{ng}$ of genomic DNA, adjusting the final volume to $25 \mu \mathrm{l}$. The PCR program started with a cycle of 3 min at $95^{\circ} \mathrm{C}$, followed by 35 cycles of denaturing $\left(30 \mathrm{~s}\right.$ at $\left.95^{\circ} \mathrm{C}\right)$, annealing $\left(45 \mathrm{~s}\right.$ at $\left.56^{\circ} \mathrm{C}\right)$, extension $\left(3 \mathrm{~min}\right.$ at $\left.72^{\circ} \mathrm{C}\right)$, and a final extension of 10 
$231 \mathrm{~min}$ at $72{ }^{\circ} \mathrm{C}$. PCR products were run on a $0.7 \%$ TAE agarose gel and were purified using the

232 Illustra GFX PCR DNA and Gel Band Purification kit (GE Healthcare).

233 The purified DNA from the PCR was used to generate FISH probes with the DecaLabel

234 DNA Labeling kit (Thermo Scientific), which is based on the random-primed method (Feinberg

235 \& Vogelstein 1983; Feinberg \& Vogelstein 1984), including a digoxigenin-labeled nucleotide.

236 The complete reaction consisted of: $10 \mu \mathrm{l}$ of decanucleotide, 5X Reaction Buffer, $1 \mu \mathrm{g}$ of cDNA,

237 and nuclease-free $\mathrm{H}_{2} \mathrm{O}$ till $42 \mu \mathrm{l}$, keeping this mix at $100^{\circ} \mathrm{C}$ for $10 \mathrm{~min}$; afterwards, we added 1

$238 \mathrm{mM}$ dNTPs mix, $1.75 \mu \mathrm{l}$ of Digoxigenin-11-dUTP (Roche), and $1 \mu 1$ of Klenow enzyme then

239 incubated at $30^{\circ} \mathrm{C}$ for 2 hours. Finally, the probes were purified again with the Illustra GFX PCR

240 DNA and Gel Band Purification kit (GE Healthcare), and eluted in $50 \mu 1$ of $\mathrm{H}_{2} \mathrm{O}$.

241 Chromosome slides were prepared from fixed gonads to observe hybridization to male

242 meiotic chromosomes from Cpe and Cpp individuals. Gonads were adhered to slides by the

243 conventional technique of squashing, and the coverslip was removed after immersing the slides

244 in liquid nitrogen. The squashed biological material was then treated for 5 min with pepsin (50

$245 \mu \mathrm{g} / \mathrm{ml}$ in $0.01 \mathrm{~N} \mathrm{HCl}$ ) at $37^{\circ} \mathrm{C}$, followed by a $30 \mathrm{~min}$ incubation in $2 \%$ paraformaldehyde at room

246 temperature. Endogenous peroxidases were inactivated by incubation for 30 min with $1 \% \mathrm{H}_{2} \mathrm{O}_{2}$.

247 Slides were then dehydrated in a series of ethanol washes $(70 \%, 85 \%$, and $100 \%)$ and dried out.

248 Slides were denatured and hybridized in the presence of $50 \mu 1$ of the hybridization mixture under

249 a coverslip for $5 \mathrm{~min}$ at $70^{\circ} \mathrm{C}$. Hybridization mixture was composed of $2 \mu 1$ of labeled probe,

$25050 \%$ formamide, $2 \mathrm{X} \mathrm{SSC}, 300 \mathrm{mM} \mathrm{NaCl}, 30 \mathrm{mM}$ sodium citrate, $\mathrm{pH}$ 7.0. After denaturing, slides

251 were left overnight in a wet chamber at $37^{\circ} \mathrm{C}$. Posthybridization washing and visualization of

252 FISH-TSA (tyramide signal amplification) probes were performed as described previously

253 (Krylov et al. 2007; Krylov et al. 2008). Detection of probes with antidigoxigenin conjugated to

254 horseradish peroxidase (Roche) was done at a concentration of 1:2000 in TNB (Tris-NaCl-

255 blocking buffer). The tyramide solution (Perkin Elmer) was incubated onto the slides for 5 min at

256 a concentration of 1:50. Chromosomes were counterstained with $50 \mathrm{ng} / \mu 1$ of DAPI (4',6-

257 diamidino-2-phenylindole, Roche) diluted in Vectashield (Vector Laboratories). Results were

258 observed in a digital image analysis platform based on Leica DMLB fluorescence microscope

259 with independent green and blue filters. Images were captured as tiff files using a cooled CCD

260 Leica DF35 monochrome camera (Leica Microsystem), and final images were processed

261 employing Photoshop CS6 (Adobe). 


\section{Data Availability}

264 All cloning and sequencing data were deposited in the GenBank database

265 (http://www.ncbi.nlm.nih.gov/genbank/) under accession numbers KR081342 - KR081347 and 266 KT599860 - KT599861. High-throughput genomic raw sequence reads are available from the 267 Sequence Read Archives (http://www.ncbi.nlm.nih.gov/sra) under BioSample accession number 268 SAMN03469681. 
269 RESULTS

270 Infected and uninfected grasshoppers across the hybrid zone harbor phage WO genes

271 To initially determine the prevalence of phage WO in the C. parallelus hybrid zone, we

272 PCR-screened hybrid, Cpe, and Cpp grasshoppers of all infection types (co-infected, B-infected,

273 F-infected and uninfected, Table S1) for the minor capsid gene ( $\operatorname{rff} 7)$, a virion structural gene

274 commonly used to identify WO haplotypes (Bordenstein \& Wernegreen 2004; Chafee et al.

275 2010; Gavotte et al. 2004; Masui et al. 2000). Surprisingly, orf7 amplicons were detected in 42

276 out of $43(98 \%)$ samples, including all uninfected grasshoppers $(n=8)$, which were determined

277 to be Wolbachia-free using nested PCR for the Wolbachia 16S ribosomal RNA gene (Figure 2).

278 Blank controls were negative for the orf7 amplicon. These results indicate that (i) phage WO is

279 or once was ubiquitous in C. parallelus and (ii) at least part of phage WO has laterally

280 transferred to the grasshopper genome.

281

282

\section{Diverse WO haplotypes are present in the grasshopper genome}

283

To identify phage WO variation in a hybrid zone population, we cloned and sequenced an

284 approximately 350 bp region of orf7 from a co-infected (604FB), B-infected (603B), F-infected

285 (607F) and uninfected (641U) hybrid grasshopper from the Portalet population (Table S2). To

286 confirm that these alleles were present in other individuals within the same population, we used

287 allele-specific primers to amplify and sequence orf7 from five additional individuals: three

288 uninfected (167U, 169U and 186U), one F-infected (180F) and one co-infected (192FB). In total,

289 we identified eight unique orf7 alleles spread throughout the phylogenetic tree of select WO

290 minor capsid sequences (Figure 3, Table S3). Seven of these alleles clustered into three

291 haplotypes (Cpar-WO1, Cpar-WO2, and Cpar-WO3) based on a 96\% identity cutoff (Figures 3

292 and 4). Since all three haplotypes contain sequences obtained from uninfected individuals, we

293 conclude that at least three phage WO insertions are present in the grasshopper nuclear genome.

294 Two alleles without an identical sequence from an uninfected individual (alleles 3 and 7) may

295 actually be present in a cytoplasmic Wolbachia strain rather than the host genome, but we have

296 conservatively clustered them within the Cpar-WO2 and Cpar-WO3 haplotypes, respectively,

297 since they are each $97.7 \%$ identical to an allele from an uninfected individual (alleles 4 and 8 ,

298 respectively). An additional orf7 allele (allele 1) was only found in a single co-infected 
299 individual, so we cannot conclude whether it was sequenced from a cytoplasmic Wolbachia 300 infection or a nuclear insert.

301 All alleles appear to be coding except for allele 7, which has a $\mathrm{C}$ to $\mathrm{T}$ substitution at

302 nucleotide 31 that introduces a premature stop codon (Figure 4). Since an identical allele was

303 identified in another individual (604FB-5), it is unlikely that the SNP is a result of a PCR or

304 sequencing error. Thus, at least one of the phage WO haplotypes may be undergoing

305 pseudogenization, which is common for Wolbachia inserts in host genomes (Brelsfoard et al.

306 2014; Nikoh et al. 2008) .

307

308 Genome sequencing reveals B and F Wolbachia DNA inserts in the grasshopper genome

The unexpected finding of intact phage WO genes in uninfected grasshoppers led us to

310 characterize the genomic inserts in the C. parallelus genome. To do so, we pooled DNA from

311 three uninfected grasshoppers from the Gabas population, which is a pure Cpp population in the

312 northern tip of the hybrid zone (Figure 1). Cpp grasshoppers were chosen for sequencing instead

313 of hybrid individuals to limit the amount of genetic variation in the sequencing and because the

314 Gabas population has a high prevalence of uninfected individuals (Zabal-Aguirre et al. 2010).

315 We used Illumina high-throughput sequencing to generate 227,349,248 paired-end reads with an

316 average length of $93.5 \mathrm{bp}$ after trimming. To extract WO reads from grasshopper sequences, we

317 first mapped all trimmed reads with a cutoff of $80 \%$ similarity over $80 \%$ read length to the

318 reference genome of the B Wolbachia strain wPip from Culex quinquefasciatus mosquitoes (Pel

319 strain, Genbank AM999887), which has five WO prophages (Klasson et al. 2008). However, in

320 addition to phage-related reads, we found that many of the 22,833 reads that mapped to $w$ Pip fell

321 outside of the WO prophage regions. Altogether, phage and non-phage Wolbachia reads covered

322 a total of $655,940 \mathrm{bp}(44 \%)$ of the $w$ Pip reference genome when non-specific reads (i.e., reads

323 with more than one match to the reference genome) were allowed to map randomly.

324 Manual observation of SNPs across the alignment revealed that many of the genes

325 appeared to have multiple alleles, some of which were more closely related to homologs in the

326 genome of F Wolbachia strain $w$ Cle [Genbank AP013028] than to those in the $w$ Pip B

327 Wolbachia strain. Indeed, phylogenetic analyses of small contigs containing portions of the dnaA

328 (Figure 5a) or $f a b G$ (Figure $5 \mathrm{~b}$ ) genes show one contig grouping with $w \mathrm{Cle}$ and the other contig

329 grouping with its homologs from strains $w$ Pip and $w$ No (both B Wolbachia strains). 
To see if the sequencing reads preferentially map to Wolbachia from supergroups other

331

332

333

334

335

336

337

338

339

340

341

342

343

344

345

346

347

348

349

350

351

352

353

354

355

356

357

358

359

360

than $\mathrm{B}$ or $\mathrm{F}$, we simultaneously mapped all reads to the $w \mathrm{Pip}, w \mathrm{Cle}, w \mathrm{Mel}, w \mathrm{Bm}$, and $w \mathrm{Oo}$ reference genomes at a cutoff of $90 \%$ sequence similarity over $90 \%$ of read length. Reads were only allowed to map exclusively to one genome, and reads that mapped ambiguously to more than one genomic location were discarded. In total, $84.7 \%$ of all mapped reads $(14,424$ out of 17,031) mapped preferentially to $w$ Pip and $w$ Cle (Table S4). A substantial number of reads $(2,517)$ totaling $74,612 \mathrm{bp}$ of the reference length also mapped to the genome of $w \mathrm{Mel}$ from the A supergroup. However, $63 \%$ of the $w$ Mel reference covered by reads (47,054 out of 74,612 bp) are annotated as mobile genetic elements like phage WO, phage-associated regions adjacent to WO and transposases. Since phage WO and other mobile elements often transfer between Wolbachia strains (Bordenstein \& Wernegreen 2004; Chafee et al. 2010; Gavotte et al. 2007; Masui et al. 2000), these phage-related reads in the grasshopper genome do not necessarily originate from an A Wolbachia genome. Furthermore, the average contig length of those contigs mapping outside of the phage regions is only $113.4 \mathrm{bp}(\mathrm{N} 50=100 \mathrm{bp})$, while contigs that map to phage and mobile elements average $229.5 \mathrm{bp}(\mathrm{N} 50=321 \mathrm{bp})$. With such short contigs in the non-phage regions, any mutational drift in the inserts due to relaxed selection could cause reads to incorrectly map to a supergroup that differed from that of the original donor.

When mapping parameters were relaxed to $65 \%$ similarity over $80 \%$ read length, the number of reads mapping to all five genomes increased considerably, although the top two genomes with the most mapped reads and longest length of reference sequence covered were still $w$ Pip and $w$ Cle (Table S4). Again, a substantial number of reads mapped to $w$ Mel but those contigs in the non-phage regions only averaged 75 bp in length $(\mathrm{N}=50)$, while those in phage regions were $228.6 \mathrm{bp}$ long an average $(\mathrm{N} 50=438)$. Likewise, contigs mapping to $w \mathrm{Bm}(\mathrm{D}$ supergroup) and wOo (C supergroup) only averaged $55 \mathrm{bp}$ and $47 \mathrm{bp}$, respectively. With longest and thus most reliable contigs mapping to $w \mathrm{Pip}, w \mathrm{Cle}$, or phage regions, we conclude that most, if not all, Wolbachia-related reads in the grasshopper genome likely transferred from either a B or F Wolbachia strain.

When all trimmed reads were mapped simultaneously to only the $w$ Pip and the $w$ Cle genomes with cutoffs of $90 \%$ sequence similarity over $90 \%$ of read length and non-specific reads mapping randomly, 14,030 reads covering 493,855 bp and 3,768 reads covering $166,490 \mathrm{bp}$ mapped to the $w$ Pip and $w$ Cle genomes, respectively (Figure 6). Together, both mappings 
361 covered a total of $660,345 \mathrm{bp}$, which is similar to the $655,940 \mathrm{bp}$ covered when mapping to $w$ Pip

362 alone at an $80 \%$ sequence similarity over $80 \%$ read length cutoff, supporting the hypothesis that

363 Wolbachia DNA in the grasshopper genome originated from both the B and F supergroups. To

364 verify that reads mapped preferentially to one supergroup over the other, reads that mapped to

365 either $w$ Pip or $w$ Cle were reciprocally mapped to the other genome with the same parameters as

366 before ( $90 \%$ sequence similarity over $90 \%$ of read length). Only $12.5 \%$ of reads that mapped to

$367 w$ Pip also mapped to $w$ Cle, while $18.6 \%$ of reads that mapped to $w$ Cle also mapped to $w$ Pip. This

368 means that, in total, $89.1 \%$ of reads $(15,332$ out of 17,798$)$ preferentially mapped to one

369 supergroup over the other. After removing the non-specific reads, the reads that preferentially

370 mapped to each genome covered approximately $448 \mathrm{~kb}$ of the $w$ Pip and $144 \mathrm{~kb}$ of the $w \mathrm{Cle}$

371 reference genomes. We note appropriate caution that this analysis does not allow us to

372 distinguish whether these are large, intact inserts or multiple smaller inserts spread throughout

373 the genome.

374 To further analyze the dual origin of the Wolbachia gene transfers, we computationally

375 searched for evidence of B and F Wolbachia inserts that contain similar genetic repertories. In

376 particular, we sought homologs in which the $w$ Pip and the $w$ Cle reference genes were both

377 covered by B- and F-specific reads of at least $80 \mathrm{bp}$. We then used blastn to verify that reads for

378 each gene homolog from one insert had a greater percent sequence similarity to $w$ Pip than to

$379 w$ Cle and vice versa. In total, we found 130 homologous genes that met these criteria (Table S5),

380 supporting a dual origin of the inserts.

381

382 Genome sequencing confirms multiple WO haplotypes in the grasshopper genome

383 Given the diversity of $\operatorname{orf} 7$ alleles sequenced from uninfected hybrid grasshoppers, it is

384 not surprising that when read coverage was mapped onto the $w$ Pip (Figure 6a) and $w$ Cle (Figure

385 6b) reference genomes, areas of higher coverage clustered mostly in the prophage regions

386 (Figure 6a). After extracting and assembling contigs from reads that mapped to the five WO

387 minor capsid (orf7) genes in $w$ Pip, we confirmed that there are at least three orf7 alleles in the

388 uninfected Cpp grasshopper genome (Figure S1). One allele (WO2-contig) is 97.3\% identical to

389 allele 4 from the Cpar-WO2 haplotype (Figure S1). The other two alleles are most similar to

390 sequences from the Cpar-WO3 haplotype: WO3-contig1 is 97.5\% identical to allele 6 and WO3-

391 contig2 is $100 \%$ identical to allele 7 (Figure S1). We did not find any orf7 alleles from the Cpar- 
392 WO1 haplotype in the genomic contigs, which may be a consequence of low sequencing 393 coverage. However, if Cpar-WO1 is absent from the Cpp genome, then it may be specific to the

394 Cpe subspecies or could even be unique to hybrids if the horizontal transfer occurred after 395 establishment of the hybrid zone.

\section{FISH localizes Wolbachia inserts in grasshopper chromosomes}

Even though, on average, $70 \%$ of individual grasshoppers from the Gabas population are uninfected with Wolbachia (Zabal-Aguirre et al. 2010), it is possible that the "uninfected" grasshoppers from Gabas had a low-titer Wolbachia infection that accounts for the sequencing of copious Wolbachia genes. This explanation is highly unlikely because PCR for two essential bacterial genes, $16 \mathrm{~S}$ rRNA and fts $Z$, failed to detect a product in all three grasshoppers pooled for sequencing, while PCR of WO genes amplified a band in all individuals for the orf7 gene (Figure S2). Moreover, to confirm Wolbachia insertions in the grasshopper genome, we used tyramidecoupled FISH to physically map Wolbachia genomic insertions in Cpe (Figure 7a) and Cpp (Figure 7b) chromosomes of uninfected male individuals. Hybridization of fluorescent DNA probes designed from a contig from the B Wolbachia insert (Table S6) revealed a discrete, repeatable distribution pattern along chromosomes in the karyotype (Figure 7), particularly in telomeric constitutive heterochromatin and in some interstitial regions. When comparing the distribution of this contig on the chromosomes of Cpp and Cpe, some signals are present at

411 homologous chromosomal locations in both genomes, such as on chromosome 4 (Figure 7, white 412 arrows), while other inserts, like that on chromosome 3 in Cpp (Figure 7, red arrows), are 413 subspecies-specific, suggesting that the former are ancestral to the last common ancestor of Cpp 414 and Cpe, whilst the latter appeared after taxon divergence. 


\section{DISCUSSION}

416 The Chorthippus parallelus hybrid zone is an excellent model for symbiosis research

417 since Wolbachia infection status is highly variable, with individuals collected at the same

418 geographical location infected with either F or B Wolbachia, co-infected with both or naturally

419 uninfected (Zabal-Aguirre et al. 2010). Though Wolbachia diversity has previously been

420 investigated in this system, this work comprises the first screen for Wolbachia's temperate phage

421 WO. We set out to characterize the types of phage WO present in the population and to

422 determine whether co-infection with Wolbachia strains from divergent B and F supergroups

423 facilitated transfer of phage WO between Wolbachia. Instead, we discovered an unexpected

424 diversity of phage WO orf7 alleles and multiple instances of horizontal transfer of the phage WO

425 orf7 gene to hybrid and non-hybrid grasshopper genomes. In total, we identified eight unique

426 orf7 alleles from nine different individuals collected from a single hybrid population. Genome

427 sequencing of Cpp grasshoppers confirmed that three of these alleles $(4,6$, and 7$)$ predate

428 secondary hybridization of Cpp and Cpe subspecies, while the other alleles may have been

429 introduced to the hybrid zone by Cpe or may be unique to hybrid populations.

430 Since many of the alleles are so similar to others ( $\geq 96 \%$ identical), they may represent

431 allelic variation at the same locus in the diploid grasshopper genome instead of independent gene

432 transfers. Thus, we conservatively classified similar alleles into three phage "haplotypes".

433 Interestingly, we did not conclusively identify orf7 alleles that were specific to Wolbachia

434 cytoplasmic infections even though many of the grasshoppers were infected by B and/or F

435 Wolbachia. It is likely that the cytoplasmic Wolbachia infections harbor phage WO with orf7

436 sequences that are so similar to those in the host genome that we cannot distinguish between the

437 two. For example, alleles 3 and 7 were only sequenced from B-infected individuals and may

438 reside in the cytoplasmic B Wolbachia genome, but further genome sequencing of the

439 cytoplasmic Wolbachia is needed to verify this observation.

$440 \quad$ After sequencing the genome of uninfected Cpp grasshopers, we discovered that not only

441 phage genes had transferred to the host genome but also large regions of both $\mathrm{B}$ and $\mathrm{F}$

442 Wolbachia. Many animal hosts that harbor or once harbored Wolbachia have evidence of

443 Wolbachia DNA in their genomes (Bordenstein 2007; Dunning Hotopp 2011; Dunning Hotopp

444 et al. 2007), probably because Wolbachia are uniquely poised for symbiont-to-host gene

445 exchange since they target the germ-line stem cell niche during host oogenesis (Fast et al. 2011; 
446 Robinson et al. 2013; Toomey et al. 2013). Wolbachia nuclear inserts can be quite large and 447 cover a substantial portion of a Wolbachia genome. For example, approximately $30 \%$ of a 448 Wolbachia genome is inserted in the X-chromosome of the bean beetle Callosobruchus chinensis 449 (Kondo et al. 2002; Nikoh et al. 2008), while an estimated $180 \mathrm{~kb}$ of Wolbachia DNA is present 450 in the genome of the longicorn beetle Monochamus alternatus (Aikawa et al. 2009). Multiple 451 Wolbachia insertions in the same host genome have also been identified. Several Drosophila 452 ananassae populations have multiple copies of an entire Wolbachia genome on one of their 453 chromosomes (Dunning Hotopp et al. 2007; Klasson et al. 2014), while the tsetse fly Glossina 454 morsitans morsitans genome has three Wolbachia chromosomal inserts with the two largest 455 inserts each covering roughly half a Wolbachia genome at $527 \mathrm{~kb}$ and $484 \mathrm{~kb}$ (Brelsfoard et al. 456 2014). The large Wolbachia inserts in this case are highly similar to each other and also closely457 related to the tsetse fly cytoplasmic Wolbachia strain, w $\mathrm{Gmm}$, suggesting a single transfer from $458 w \mathrm{Gmm}$ to the tsetse fly genome followed by duplication of the insert, though independent 459 transfer events cannot be ruled out (Brelsfoard et al. 2014). Either way, both insertions came 460 from the same Wolbachia supergroup and likely from the same Wolbachia strain.

461 In our study, phylogenetic analyses of variable contigs mapping to the same Wolbachia 462 genes revealed that inserts in the $C$. parallelus genome likely originated from both $\mathrm{B}$ and $\mathrm{F}$ 463 Wolbachia. To our knowledge, this is the first case of substantial Wolbachia DNA transfer from 464 divergent supergroups into the same host genome. Similar techniques used to analyze the 465 genomes of Wolbachia-free nematodes such as Acanthocheilonema viteae, Onchocerca flexuosa, 466 Loa loa, and Dictyocaulus viviparus found ancient remnants of Wolbachia genes that appear to 467 have originated from multiple supergroups when compared to present-day cytoplasmic 468 Wolbachia genes (Desjardins et al. 2013; Koutsovoulos et al. 2014; McNulty et al. 2010). 469 However, the antiquity of these horizontal transfer events makes accurate phylogenetic 470 inferences difficult, especially since the Wolbachia genes in the nematode host are no longer 471 under the same selective pressures as cytoplasmic Wolbachia genes. For example, McNulty et al. 4722010 estimates that "fossilized" evidence of Wolbachia sequences in the genomes of $A$. viteae 473 and $O$. flexuosa must be several million years old based on their low percent identities (78\% and $47481 \%$, respectively) to any contemporary Wolbachia sequences. In contrast, average percent 475 identities of the B Wolbachia gene variants to $w$ Pip and the F Wolbachia gene variants to $w$ Cle 
476 for the 130 shared genes in the C. parallelus inserts (Table S5) are $94 \pm 0.05 \%$ and $93 \pm 0.04 \%$, 477 respectively.

478 The higher percent identity to a contemporary Wolbachia strain for the grasshopper

479 inserts suggest that they have transferred more recently and/or are better preserved in the

480 grasshopper genome due to the unique evolutionary dynamics of grasshopper genomes.

481 Orthopterans like grasshoppers, locusts and crickets are known for their enormous genomes, and

482 C. parallelus grasshoppers have one of the largest genomes in the order with estimates ranging

483 from 12.3 to $14.7 \mathrm{~Gb}$ (Lechner et al. 2013). Genome gigantism in Orthoptera is thought to

484 largely be due to frequent acquisition of new genetic material coupled with slow rates of DNA

485 loss (Bensasson et al. 2001; Bensasson et al. 2000; Song et al. 2014). For example, Orthopteran

486 genomes exhibit unusually high rates of DNA transfer from mitochondria to the nuclear genome

487 (Bensasson et al. 2000; Song et al. 2014), and, with the slow rate of DNA loss, some of these

488 inserts have remained intact for 150 million years (Song et al. 2014). Based on the rate of

489 mitochondrial gene acquisition, grasshopper genomes may be more amenable to horizontal gene

490 transfer in general, especially from intracellular cytoplasmic entities like mitochondria or

491 Wolbachia. It is not surprising, then, to presume that DNA from both B and F Wolbachia would

492 eventually wind up in the C. parallelus host genome.

493 The dynamic nature of Wolbachia lateral gene transfer to the C. parallelus genome is

494 evident when visualized with FISH. Some inserts are present at the same position on the

495 chromosomes of both Cpp and Cpe while other inserts are subspecies-specific, indicating that

496 insertion events likely occurred both before and after the divergence of the subspecies. Our

497 sequencing of the WO orf7 gene supports this hypothesis since the Cpar-WO2 and Cpar-WO3

498 haplotypes are present in the genomes of Cpp individuals from Gabas and in hybrids from

499 Portalet, while the Cpar-WO1 haplotype was only detected in Portalet. Subspecies-specific

500 sequences are likely relatively young since the two subspecies are estimated to have diverged

501 between 0.2 and 2 MYA (Cooper \& Hewitt 1993; Lunt et al. 1998). If hybrid-specific inserts

502 arose independently, they would be even younger since the transfer would have had to occur

503 after the formation of the hybrid zone roughly 9,000 years ago (Hewitt 1993; Shuker et al.

504 2005a). Thus, slow rates of DNA loss coupled with relatively recent transfer events allows

505 standard phylogenetic analyses to easily identify and distinguish the inserts in the C. parallelus 
506 genome as originating from either a B or F Wolbachia, whereas Wolbachia inserts in nematode 507 genomes may be too divergent to accurately predict the donor Wolbachia's supergroup.

508 Instead of independent transfers, B and F Wolbachia strains may have recombined to 509 produce a Wolbachia strain with genes from both supergroups and part of this "hybrid"

510 Wolbachia genome transferred as a single event into the C. parallelus genome. This scenario 511 appears unlikely as we identified 130 Wolbachia genes with multiple alleles from both B and F

512 Wolbachia in the genomic inserts. A recombinogenic genome with substantial genetic

513 redundancy of essential genes is improbable given that endosymbiont genomes tend to be

514 relatively streamlined (Newton \& Bordenstein 2011; Wernegreen 2002). Furthermore, FISH 515 analyses verified the presence of Wolbachia DNA in multiple locations on the C.parallelus

516 chromosomes and further characterization of the inserts and their evolutionary history is in 517 progress. 


\section{CONCLUSION}

Alongside genetic introgression, animal hybrid zones offer an avenue for symbiont exchange, especially for heritable endosymbionts like Wolbachia (Mandel et al. 2001; Zabal-

521 Aguirre et al. 2010). Resulting co-infections of multiple Wolbachia strains in a hybrid host

522 provide opportunities for genetic exchange within the intracellular arena (Bordenstein \&

523 Reznikoff 2005; Metcalf \& Bordenstein 2012; Newton \& Bordenstein 2011). Though exchange

524 of bacteriophage WO occurs often between co-infections of A and B Wolbachia (Bordenstein \&

525 Wernegreen 2004; Chafee et al. 2010; Kent et al. 2011; Masui et al. 2000), we found no evidence

526 for phage WO transfer among B and F Wolbachia in hybrid C. parallelus grasshoppers. Instead,

527 we found that horizontal gene transfer is clearly a dynamic process in C. parallelus, with two

528 discrete Wolbachia supergroups (B and F) transferring approximately $448 \mathrm{~kb}$ and $144 \mathrm{~kb}$ of

529 DNA, respectively, to the host genome. Since many insects are co-infected with Wolbachia from

530 different supergroups, it is curious why there are not more insect genomes with Wolbachia

531 inserts of dual origin. Part of the answer is likely that other genomes with inserts of dual origin

532 have simply not been sequenced yet. However, grasshopper and other Orthopteran genomes,

533 with their high rates of DNA acquisition and slow rates of DNA loss, may be uniquely poised for

534 acquiring Wolbachia genes and maintaining them relatively intact for long periods of time,

535 allowing phylogenetic analyses to accurately distinguish between different supergroups. Though

536 the gigantic genomes of Orthopterans currently make them challenging to sequence and

537 assemble, it will be interesting to see if more species of this undersampled insect order also have

538 DNA from multiple endosymbionts in their genomes. 


\section{ACKNOWLEDGEMENTS}

540 We thank Sarah Bordenstein for advice on the project. We are grateful to the Spanish

541 Comunidad de Madrid, the Gobierno de Aragón and the French Parc National des Pyrénées for

542 permission to collect grasshoppers. We thank Prof. J.M. Szymura for orientating us to the

543 Tyramide-coupled FISH to detect small DNA sequences and to Prof. V. Krylov for his advice to 544 set up this technique.

545 
546

547

548

549

550

551

552

553

554

555

556

557

558

559

560

561

562

563

564

565

566

567

568

569

570

571

572

573

574

575

576

577

578

579

580

581

582

583

584

585

586

587

588

589

\section{REFERENCES}

Aikawa T, Anbutsu H, Nikoh N, Kikuchi T, Shibata F, and Fukatsu T. 2009. Longicorn beetle that vectors pinewood nematode carries many Wolbachia genes on an autosome. Proceedings Biological sciences / The Royal Society 276:3791-3798. 10.1098/rspb.2009.1022

Alikhan NF, Petty NK, Ben Zakour NL, and Beatson SA. 2011. BLAST Ring Image Generator (BRIG): simple prokaryote genome comparisons. BMC genomics 12:402. 10.1186/1471-2164-12-402

Bella JL, Hewitt GM, and Gosalvez J. 1990. Meiotic imbalance in laboratory produced hybrid males of Chorthippus parallelus parallelus and Chorthippus parallelus erythropus. Genetics Research 56:43-48.

Bella JL, Serrano L, Orellana J, and Mason PL. 2007. The origin of the Chorthippus parallelus hybrid zone: chromosomal evidence of multiple refugia for Iberian populations. Journal of evolutionary biology 20:568-576. 10.1111/j.1420-9101.2006.01254.x

Bensasson D, Petrov DA, Zhang DX, Hartl DL, and Hewitt GM. 2001. Genomic gigantism: DNA loss is slow in mountain grasshoppers. Molecular biology and evolution 18:246253.

Bensasson D, Zhang DX, and Hewitt GM. 2000. Frequent assimilation of mitochondrial DNA by grasshopper nuclear genomes. Molecular biology and evolution 17:406-415.

Bordenstein SR. 2007. Evolutionary genomics: transdomain gene transfers. Current Biology 17:R935-936. 10.1016/j.cub.2007.09.022

Bordenstein SR, and Reznikoff WS. 2005. Mobile DNA in obligate intracellular bacteria. Nature reviews Microbiology 3:688-699. 10.1038/nrmicro1233

Bordenstein SR, and Wernegreen JJ. 2004. Bacteriophage flux in endosymbionts (Wolbachia): infection frequency, lateral transfer, and recombination rates. Molecular biology and evolution 21:1981-1991. 10.1093/molbev/msh211

Brelsfoard C, Tsiamis G, Falchetto M, Gomulski LM, Telleria E, Alam U, Doudoumis V, Scolari F, Benoit JB, Swain M, Takac P, Malacrida AR, Bourtzis K, and Aksoy S. 2014. Presence of extensive Wolbachia symbiont insertions discovered in the genome of its host Glossina morsitans morsitans. PLoS neglected tropical diseases 8:e2728. 10.1371/journal.pntd.0002728

Casiraghi M, Anderson TJ, Bandi C, Bazzocchi C, and Genchi C. 2001. A phylogenetic analysis of filarial nematodes: comparison with the phylogeny of Wolbachia endosymbionts. Parasitology 122 Pt 1:93-103.

Chafee ME, Funk DJ, Harrison RG, and Bordenstein SR. 2010. Lateral phage transfer in obligate intracellular bacteria (wolbachia): verification from natural populations. Molecular biology and evolution 27:501-505. 10.1093/molbev/msp275

Cooper SJ, and Hewitt GM. 1993. Nuclear DNA sequence divergence between parapatric subspecies of the grasshopper Chorthippus parallelus. Insect molecular biology 2:185-194.

Desjardins CA, Cerqueira GC, Goldberg JM, Dunning Hotopp JC, Haas BJ, Zucker J, Ribeiro JM, Saif S, Levin JZ, Fan L, Zeng Q, Russ C, Wortman JR, Fink DL, Birren BW, and Nutman TB. 2013. Genomics of Loa loa, a Wolbachia-free filarial parasite of humans. Nature Genetics 45:495-500. 10.1038/ng.2585 
590

591

592

593

594

595

596

597

598

599

600

601

602

603

604

605

606

607

608

609

610

611

612

613

614

615

616

617

618

619

620

621

622

623

624

625

626

627

628

629

630

631

632

633

634

Douglas AE. 2011. Lessons from studying insect symbioses. Cell Host Microbe 10:359-367. 10.1016/j.chom.2011.09.001

Dunning Hotopp JC. 2011. Horizontal gene transfer between bacteria and animals. Trends in genetics : TIG 27:157-163.10.1016/j.tig.2011.01.005

Dunning Hotopp JC, Clark ME, Oliveira DC, Foster JM, Fischer P, Munoz Torres MC, Giebel JD, Kumar N, Ishmael N, Wang S, Ingram J, Nene RV, Shepard J, Tomkins J, Richards S, Spiro DJ, Ghedin E, Slatko BE, Tettelin H, and Werren JH. 2007. Widespread lateral gene transfer from intracellular bacteria to multicellular eukaryotes. Science 317:1753-1756. 10.1126/science.1142490

Fast EM, Toomey ME, Panaram K, Desjardins D, Kolaczyk ED, and Frydman HM. 2011. Wolbachia enhance Drosophila stem cell proliferation and target the germline stem cell niche. Science 334:990-992. 10.1126/science.1209609

Feinberg AP, and Vogelstein B. 1983. A technique for radiolabeling DNA restriction endonuclease fragments to high specific activity. Anal Biochem 132:6-13.

Feinberg AP, and Vogelstein B. 1984. "A technique for radiolabeling DNA restriction endonuclease fragments to high specific activity". Addendum. Anal Biochem 137:266-267.

Gavotte L, Henri H, Stouthamer R, Charif D, Charlat S, Bouletreau M, and Vavre F. 2007. A Survey of the bacteriophage WO in the endosymbiotic bacteria Wolbachia. Molecular biology and evolution 24:427-435. 10.1093/molbev/msl171

Gavotte L, Vavre F, Henri H, Ravallec M, Stouthamer R, and Bouletreau M. 2004. Diversity, distribution and specificity of WO phage infection in Wolbachia of four insect species. Insect molecular biology 13:147-153. 10.1111/j.0962-1075.2004.00471.x

Guindon S, and Gascuel 0. 2003. A simple, fast, and accurate algorithm to estimate large phylogenies by maximum likelihood. Systematic Biology 52:696-704.

Harrison RG, and Arnold J. 1982. A narrow hybrid zone between closely related cricket species. Evolution; international journal of organic evolution 36:535-552.

Hasegawa M, Kishino $\mathrm{H}$, and Yano T. 1985. Dating of the human-ape splitting by a molecular clock of mitochondrial DNA. Journal of molecular evolution 22:160-174.

Hewitt GM. 1993. After the ice: parallelus meets erythropus in the Pyrenees. In: Harrison RG, ed. Hybrid Zones and the Evolutionary Process. Oxford: Oxford University Press, 140-164.

Huelsenbeck JP, and Ronquist F. 2001. MRBAYES: Bayesian inference of phylogenetic trees. Bioinformatics 17:754-755.

Jaenike J, Dyer KA, Cornish C, and Minhas MS. 2006. Asymmetrical reinforcement and Wolbachia infection in Drosophila. PLoS biology 4:e325. 10.1371/journal.pbio.0040325

Kent BN, Salichos L, Gibbons JG, Rokas A, Newton IL, Clark ME, and Bordenstein SR. 2011. Complete bacteriophage transfer in a bacterial endosymbiont (Wolbachia) determined by targeted genome capture. Genome biology and evolution 3:209-218. 10.1093/gbe/evr007

Klasson L, Kumar N, Bromley R, Sieber K, Flowers M, Ott SH, Tallon LJ, Andersson SG, and Dunning Hotopp JC. 2014. Extensive duplication of the Wolbachia DNA in chromosome four of Drosophila ananassae. BMC genomics 15:1097. 10.1186/14712164-15-1097 
635 Klasson L, Walker T, Sebaihia M, Sanders MJ, Quail MA, Lord A, Sanders S, Earl J, O'Neill SL,

636

637

638

639

640

641

642

643

644

645

646

647

648

649

650

651

652

653

654

655

656

657

658

659

660

661

662

663

664

665

666

667

668

669

670

671

672

673

674

675

676

677

678

679

680 Thomson N, Sinkins SP, and Parkhill J. 2008. Genome evolution of Wolbachia strain wPip from the Culex pipiens group. Molecular biology and evolution 25:1877-1887. $10.1093 / \mathrm{molbev} / \mathrm{msn} 133$

Kondo N, Nikoh N, Ijichi N, Shimada M, and Fukatsu T. 2002. Genome fragment of Wolbachia endosymbiont transferred to X chromosome of host insect. Proceedings of the National Academy of Sciences of the United States of America 99:14280-14285. 10.1073/pnas.222228199

Koutsovoulos G, Makepeace B, Tanya VN, and Blaxter M. 2014. Palaeosymbiosis revealed by genomic fossils of Wolbachia in a strongyloidean nematode. PLoS genetics 10:e1004397. 10.1371/journal.pgen.1004397

Krylov V, Tlapakova T, and Macha J. 2007. Localization of the single copy gene Mdh2 on Xenopus tropicalis chromosomes by FISH-TSA. Cytogenet Genome Res 116:110-112. $10.1159 / 000097427$

Krylov V, Tlapakova T, Macha J, Curlej J, Ryban L, and Chrenek P. 2008. Localization of human coagulation factor VIII (hFVIII) in transgenic rabbit by FISH-TSA: identification of transgene copy number and transmission to the next generation. Folia Biol (Praha) 54:121-124.

Lanave C, Preparata G, Saccone C, and Serio G. 1984. A new method for calculating evolutionary substitution rates. Journal of molecular evolution 20:86-93.

Lechner M, Marz M, Ihling C, Sinz A, Stadler PF, and Krauss V. 2013. The correlation of genome size and DNA methylation rate in metazoans. Theory in biosciences $=$ Theorie in den Biowissenschaften 132:47-60. 10.1007/s12064-012-0167-y

LePage D, and Bordenstein SR. 2013. Wolbachia: Can we save lives with a great pandemic? Trends in Parasitology 29:385-393. 10.1016/j.pt.2013.06.003

Lo N, Casiraghi M, Salati E, Bazzocchi C, and Bandi C. 2002. How many wolbachia supergroups exist? Molecular biology and evolution 19:341-346.

Lunt DH, Ibrahim KM, and Hewitt GM. 1998. mtDNA phylogeography and postglacial patterns of subdivision in the meadow grasshopper Chorthippus parallelus. Heredity 80 ( Pt 5):633-641.

Mandel MJ, Ross CL, and Harrison RG. 2001. Do Wolbachia infections play a role in unidirectional incompatibilities in a field cricket hybrid zone? Molecular ecology 10:703-709.

Martinez-Rodriguez P, Hernandez-Perez M, and Bella JL. 2013. Detection of Spiroplasma and Wolbachia in the bacterial gonad community of Chorthippus parallelus. Microb Ecol 66:211-223. 10.1007/s00248-013-0226-z

Masui S, Kamoda S, Sasaki T, and Ishikawa H. 2000. Distribution and evolution of bacteriophage WO in Wolbachia, the endosymbiont causing sexual alterations in arthropods. Journal of molecular evolution 51:491-497.

Masui S, Kuroiwa H, Sasaki T, Inui M, Kuroiwa T, and Ishikawa H. 2001. Bacteriophage WO and virus-like particles in Wolbachia, an endosymbiont of arthropods. Biochemical and biophysical research communications 283:1099-1104. 10.1006/bbrc.2001.4906

McNulty SN, Foster JM, Mitreva M, Dunning Hotopp JC, Martin J, Fischer K, Wu B, Davis PJ, Kumar S, Brattig NW, Slatko BE, Weil GJ, and Fischer PU. 2010. Endosymbiont DNA in endobacteria-free filarial nematodes indicates ancient horizontal genetic transfer. PloS one 5:e11029. 10.1371/journal.pone.0011029 
681

682

683

684

685

686

687

688

689

690

691

692

693

694

695

696

697

698

699

700

701

702

703

704

705

706

707

708

709

710

711

712

713

714

715

716

717

718

719

720

721

722

723

724

725

726

Metcalf JA, and Bordenstein SR. 2012. The complexity of virus systems: the case of endosymbionts. Current Opinion Microbiology 15:546-552. 10.1016/j.mib.2012.04.010

Newton IL, and Bordenstein SR. 2011. Correlations between bacterial ecology and mobile DNA. Current microbiology 62:198-208. 10.1007/s00284-010-9693-3

Nikoh N, Tanaka K, Shibata F, Kondo N, Hizume M, Shimada M, and Fukatsu T. 2008. Wolbachia genome integrated in an insect chromosome: evolution and fate of laterally transferred endosymbiont genes. Genome research 18:272-280. 10.1101/gr.7144908

Pais R, Lohs C, Wu Y, Wang J, and Aksoy S. 2008. The obligate mutualist Wigglesworthia glossinidia influences reproduction, digestion, and immunity processes of its host, the tsetse fly. Applied and environmental microbiology 74:5965-5974. 10.1128/AEM.00741-08

Robinson KM, Sieber KB, and Dunning Hotopp JC. 2013. A review of bacteria-animal lateral gene transfer may inform our understanding of diseases like cancer. PLoS genetics 9:e1003877. 10.1371/journal.pgen.1003877

Serbus LR, Casper-Lindley C, Landmann F, and Sullivan W. 2008. The genetics and cell biology of Wolbachia-host interactions. Annual Review of Genetics 42:683-707. 10.1146/annurev.genet.41.110306.130354

Shoemaker DD, Katju V, and Jaenike J. 1999. Wolbachia and the evolution of reproductive isolation between Drosophila recens and Drosophila subquinaria. Evolution; international journal of organic evolution 53:1157-1164.

Shuker DM, King TM, Bella JL, and Butlin RK. 2005a. The genetic basis of speciation in a grasshopper hybrid zone. In: Fellowes M, Holloway G, and Roff J, eds. Insect Evolutionary Biology. Wallingford, Oxon, UK: CABI Publishing, Oxford University Press, 427-454.

Shuker DM, Underwood K, King TM, and Butlin RK. 2005b. Patterns of male sterility in a grasshopper hybrid zone imply accumulation of hybrid incompatibilities without selection. Proceedings Biological sciences / The Royal Society 272:2491-2497. 10.1098/rspb.2005.3242

Song H, Moulton MJ, and Whiting MF. 2014. Rampant nuclear insertion of mtDNA across diverse lineages within Orthoptera (Insecta). PloS one 9:e110508. 10.1371/journal.pone.0110508

Tamas I, Klasson L, Canback B, Naslund AK, Eriksson AS, Wernegreen JJ, Sandstrom JP, Moran NA, and Andersson SG. 2002. 50 million years of genomic stasis in endosymbiotic bacteria. Science 296:2376-2379. 10.1126/science.1071278

Tavare S. 1986. Some probabilistic and statistical problems in the analysis of DNA sequences. Lecture Notes on Mathematical Modelling in the Life Sciences 17:57-86.

Toomey ME, Panaram K, Fast EM, Beatty C, and Frydman HM. 2013. Evolutionarily conserved Wolbachia-encoded factors control pattern of stem-cell niche tropism in Drosophila ovaries and favor infection. Proceedings of the National Academy of Sciences of the United States of America 110:10788-10793. 10.1073/pnas. 1301524110

van Ham RC, Kamerbeek J, Palacios C, Rausell C, Abascal F, Bastolla U, Fernandez JM, Jimenez L, Postigo M, Silva FJ, Tamames J, Viguera E, Latorre A, Valencia A, Moran F, and Moya A. 2003. Reductive genome evolution in Buchnera aphidicola. Proceedings

PeerJ reviewing PDF | (2015:09:6642:1:0:NEW 9 Nov 2015) 
727

728

729

730

731

732

733

734

735

736

737

738

739

740

741

742

743

744

745

of the National Academy of Sciences of the United States of America 100:581-586. 10.1073/pnas.0235981100

Weinert LA, Araujo-Jnr EV, Ahmed MZ, and Welch JJ. 2015. The incidence of bacterial endosymbionts in terrestrial arthropods. Proceedings Biological sciences / The Royal Society 282:20150249. 10.1098/rspb.2015.0249

Wernegreen JJ. 2002. Genome evolution in bacterial endosymbionts of insects. Nature Reviews Genetics 3:850-861. 10.1038/nrg931

Zabal-Aguirre M, Arroyo F, and Bella JL. 2010. Distribution of Wolbachia infection in Chorthippus parallelus populations within and beyond a Pyrenean hybrid zone. Heredity 104:174-184. 10.1038/hdy.2009.106

Zabal-Aguirre M, Arroyo F, Garcia-Hurtado J, de la Torre J, Hewitt GM, and Bella JL. 2014. Wolbachia effects in natural populations of Chorthippus parallelus from the Pyrenean hybrid zone. Journal of evolutionary biology 27:1136-1148. 10.1111/jeb.12389

Zug R, and Hammerstein P. 2012. Still a host of hosts for Wolbachia: analysis of recent data suggests that $40 \%$ of terrestrial arthropod species are infected. PloS one 7:e38544. 10.1371/journal.pone.0038544 


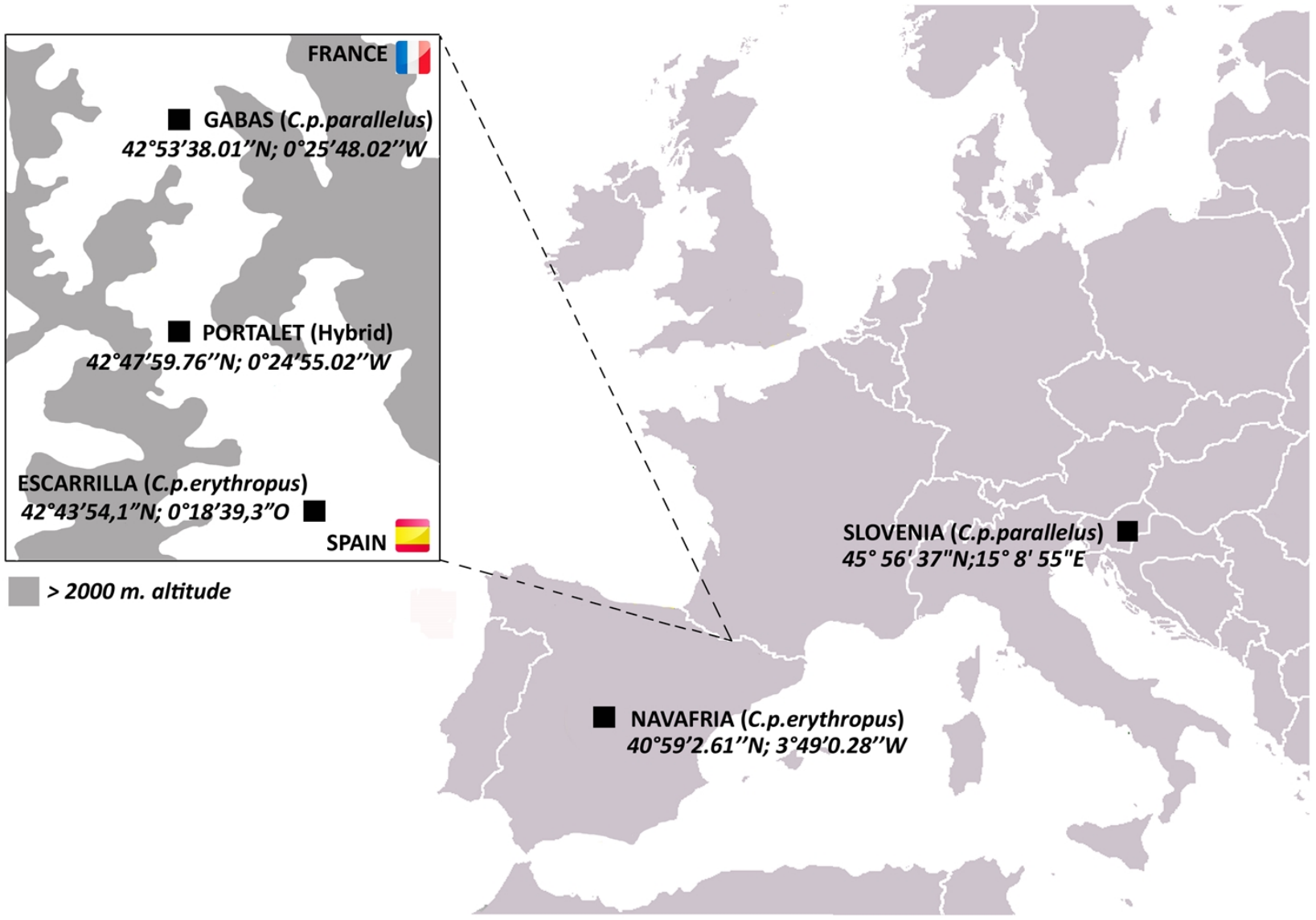

747

748 Figure 1. Map of $\boldsymbol{C}$. parallelus collection sites with their geographical coordinates. Boxed

749 inset shows the hybrid zone of C. p. parallelus and C. p. erythropus subspecies in the D'Ossau

750 and Tena valleys of the Pyrenees Mountains between France and Spain. 
752

753

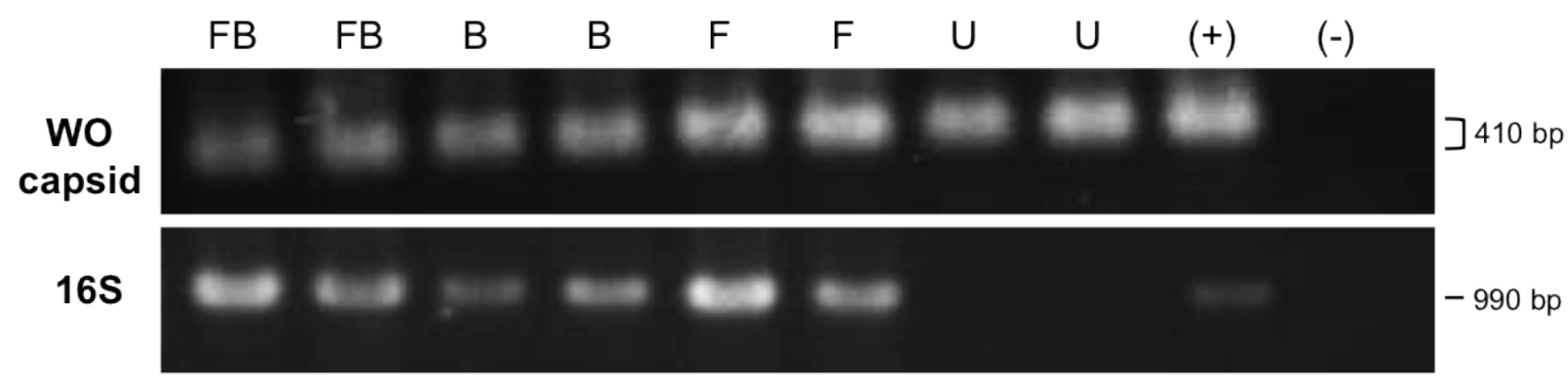

754 Figure 2. PCR amplification of the WO minor capsid (orf 7 ) gene (top) and 16S ribosomal

755 RNA gene (bottom). Two individuals of each infection type are shown: $\mathrm{FB}=$ co-infected, $\mathrm{B}=\mathrm{B}$

756 infection only, $\mathrm{F}=\mathrm{F}$ infection only, $\mathrm{U}=$ uninfected, $(+)=$ positive DNA control, $(-)=$ no

757 template negative control. For the WO capsid gene, the gel ran askew, making some bands

758 appear larger in size than others though all bands represent the same sized PCR amplicon (410

759 bp).

760 


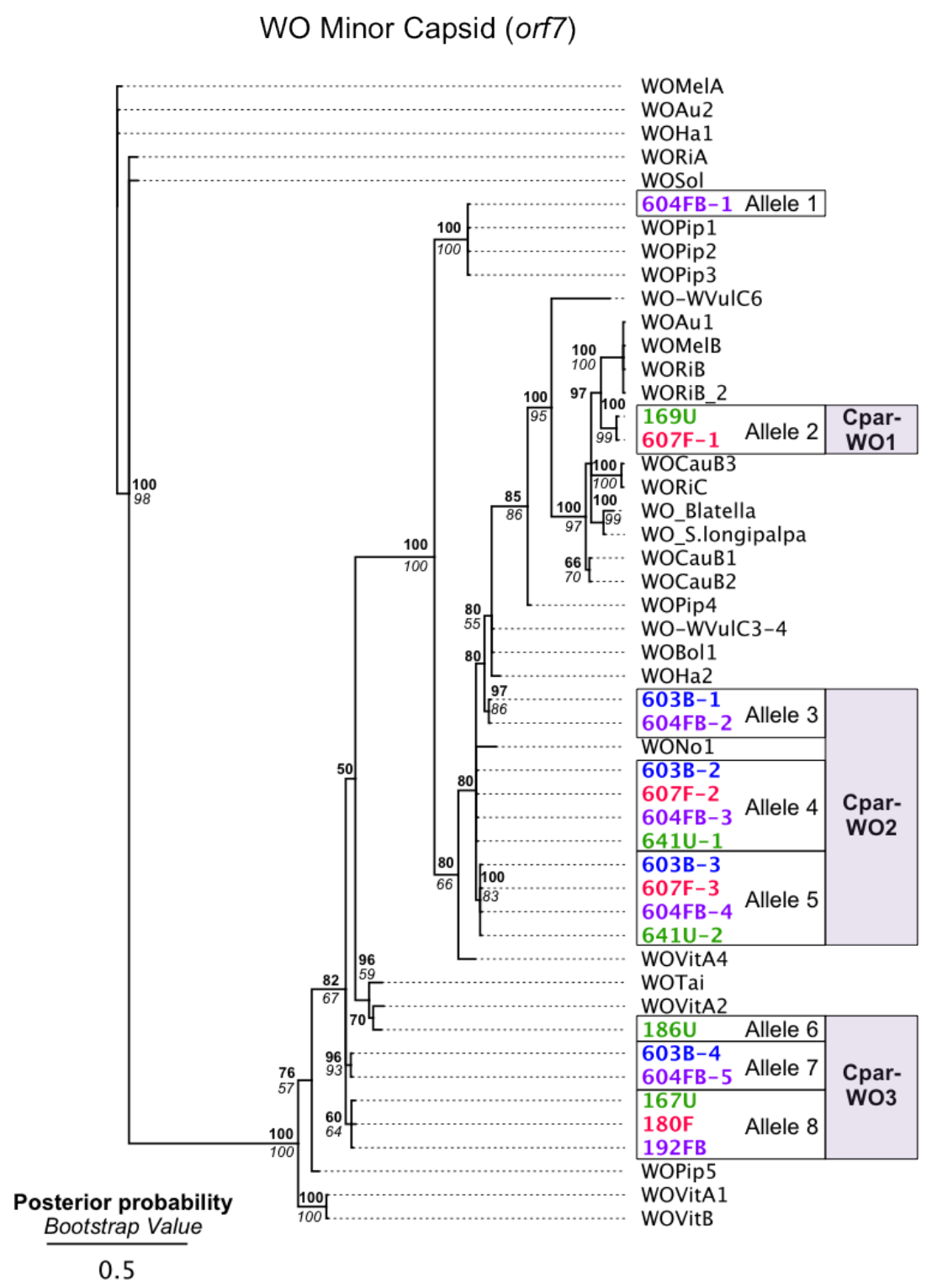

761

762 Figure 3. Phylogeny of the WO minor capsid (orf 7 ) gene. Bayesian phylogeny constructed

763 using indel-free nucleotide alignment of the phage WO orf7 gene. Sequences generated in this

764 study are labeled with individual identification numbers and color-coded based on the

765 grasshopper's infection status: $\mathrm{FB}=$ co-infected (purple), $\mathrm{B}=\mathrm{B}$-infection only (blue), $\mathrm{F}=\mathrm{F}$ -

766 infection only (red) and $\mathrm{U}=$ uninfected (green). Numbers after a hyphen designate different orf7

767 sequences from the same individual. Posterior probability (Bayesian) and bootstrap (maximum

768 likelihood) values over 50 are indicated in bold and italics, respectively. Accession numbers for

769 sequences used in the tree, including the sequences from this study, are listed in Table S3. The

770 tree is arbitrarily rooted. 
WO Minor Capsid (orf7)

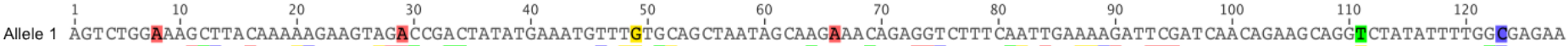

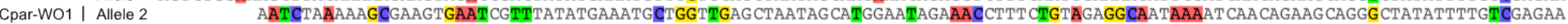

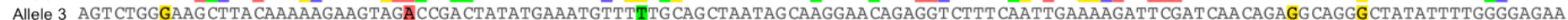

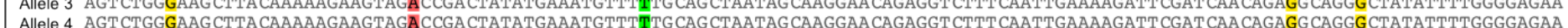

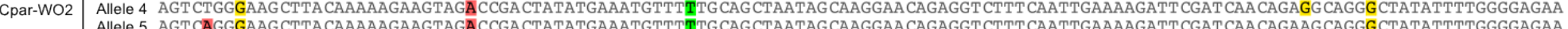

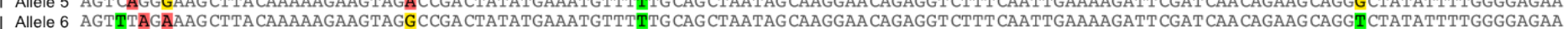

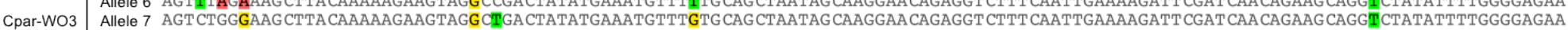

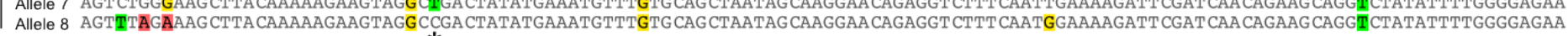

$$
130
$$
140 150 160 170 190 200 210 220 230 240 250

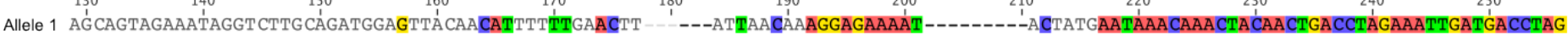
Cpar-WO1 | Allele 2 AGCAGTAGAAATAGGTCTTGCAGATGGAATTACAATTCTTTCAGAGTTTAAATCTATTAATAAAAAC Allele 3 AGCAGTAGAAATAGGCCTTGCAGACGGAATGA CAATTCTTTCA------ TCTATTAATAAAAA

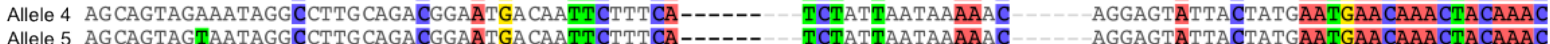
Allele 5 AGCAGTAGTAATAGGCCTTGCAGACGGATGACAATTCTTTCA-----

Cpar-WO3 $\quad$ Allele 7 AGCAGTAGAAATAGGTCTTGCAGATGGAGTTACAACATTTTTTGAATTT Allele 8 AGCAGTAGAAATAGGT CTTGCAGATGGAGTTA CAACATTTTTTGAATTT TCTATTAATAAAAA - - ATCATAATCATAGGAGTGTTAGTATG
260
270
300
$310 \quad 320$
330
340
$350 \quad 353$

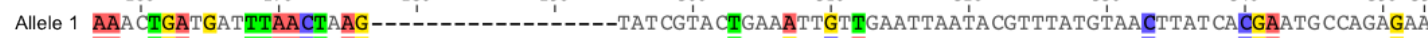
Allele 2 AAA CTGATAATTTAACTAAG-----------TAT CGTA CTGAAGTT CT AGAATTAATA CGTTTATGTAACTTAT CACGAATG CCAGAGAA Allele 3 AAA CTGATAATTTAACCAAG-------------TAT CGTACTGAAGTT TTT TAAATTAATA CGTTTATGTAATTTAT CGAAGGTGCCAGAAAA Allele 4 AAA CTGATAATTTAACTAAG-------------TAT CGTA CTGAAGTTCTTGAATTAATA CGTTTATGTAACTTAT CACGAATG CCAGAAAA

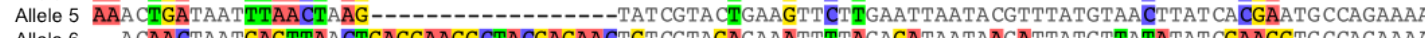
Allele 6 --ACAACTAATGAGTTAACTGAGGAAGGCTACGAGAACTGT CGTAGAGAAATTTTÄGAGATAATAAGATTATGTTATATAT CGAAGGTGCCAGAAAA

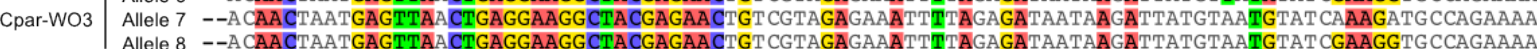
772

773 Figure 4. Nucleotide alignment of WO minor capsid (orf7) alleles from hybrid

774 grasshoppers. Asterisk indicates location of $\mathrm{C}$ to $\mathrm{T}$ substitution that introduces a premature stop

775 codon in Cpar-WO3, allele 7. Nucleotides are counted from the start of the sequence alignment,

776 not from the transcription start site of the gene.

777 


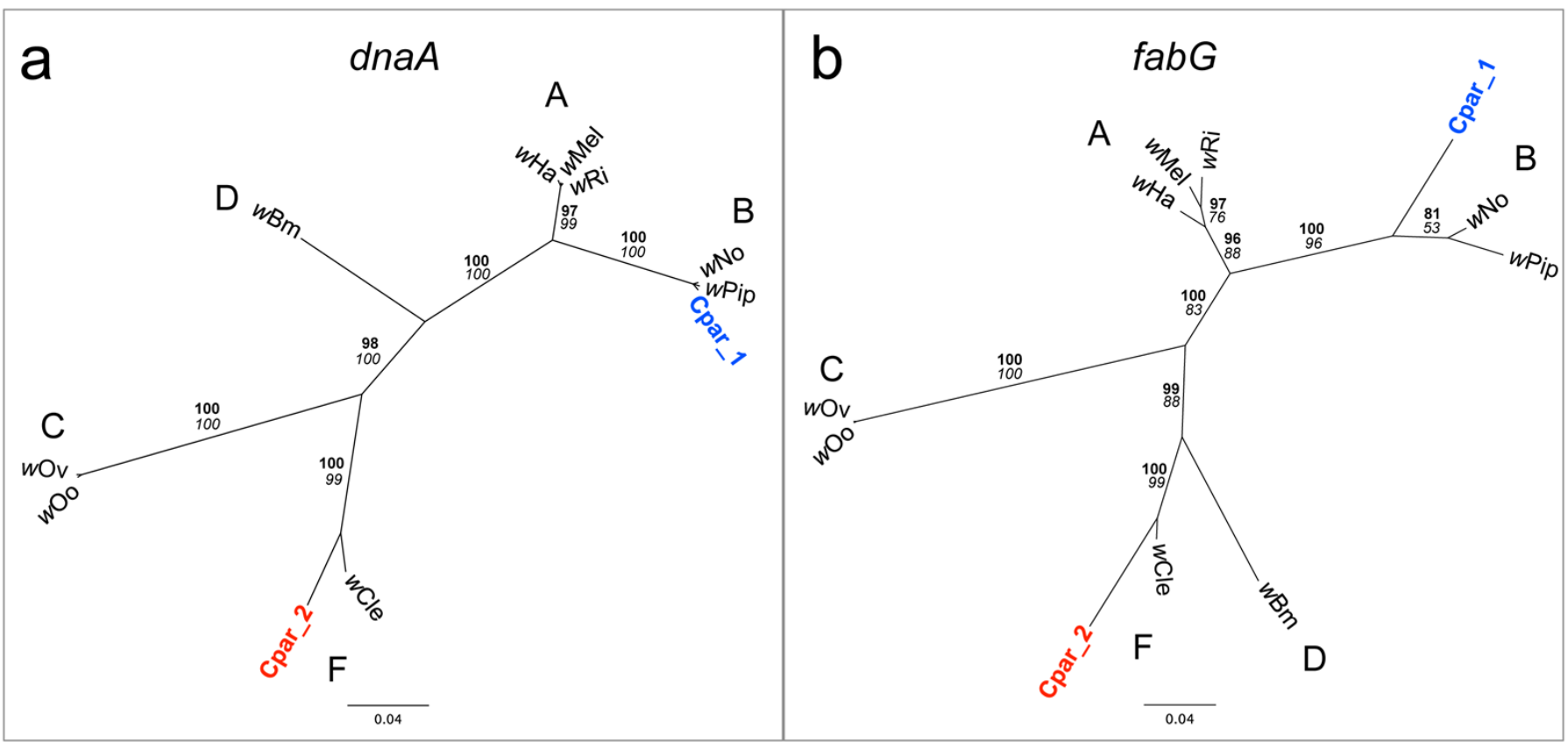

780 Figure 5. Phylogenies of Wolbachia dnaA and fabG genes with . parallelus genomic inserts.

781 Unrooted Bayesian phylogenies constructed using indel-free nucleotide alignments of Wolbachia

782 (a) dnaA and (b) $f a b G$ genes with homologous contigs from C. parallelus genomic inserts (blue 783 and red labels). Wolbachia supergroups (A-D, F) are indicated next to their respective clades.

784 Posterior probability (bold) and bootstrap (italicized) values over 50 are indicated at each branch.

785 Sequences for $d n a A$ and $f a b G$ genes were extracted from the full genome sequences of their

786 respective Wolbachia from NCBI (Genbank) as follows: $w \mathrm{Ha}$ [CP003884.1], wMel

787 [AE017196.1], $w$ Ri [CP001391.1], $w$ No [CP003883.1], $w$ Pip strain Pel [AM999887.1], $w$ Oo

788 [HE660029.1], wOv strain Cameroon [HG810405.1], $w$ Bm strain TRS [AE017321.1], and $w$ Cle 789 [AP013028.1].

790 


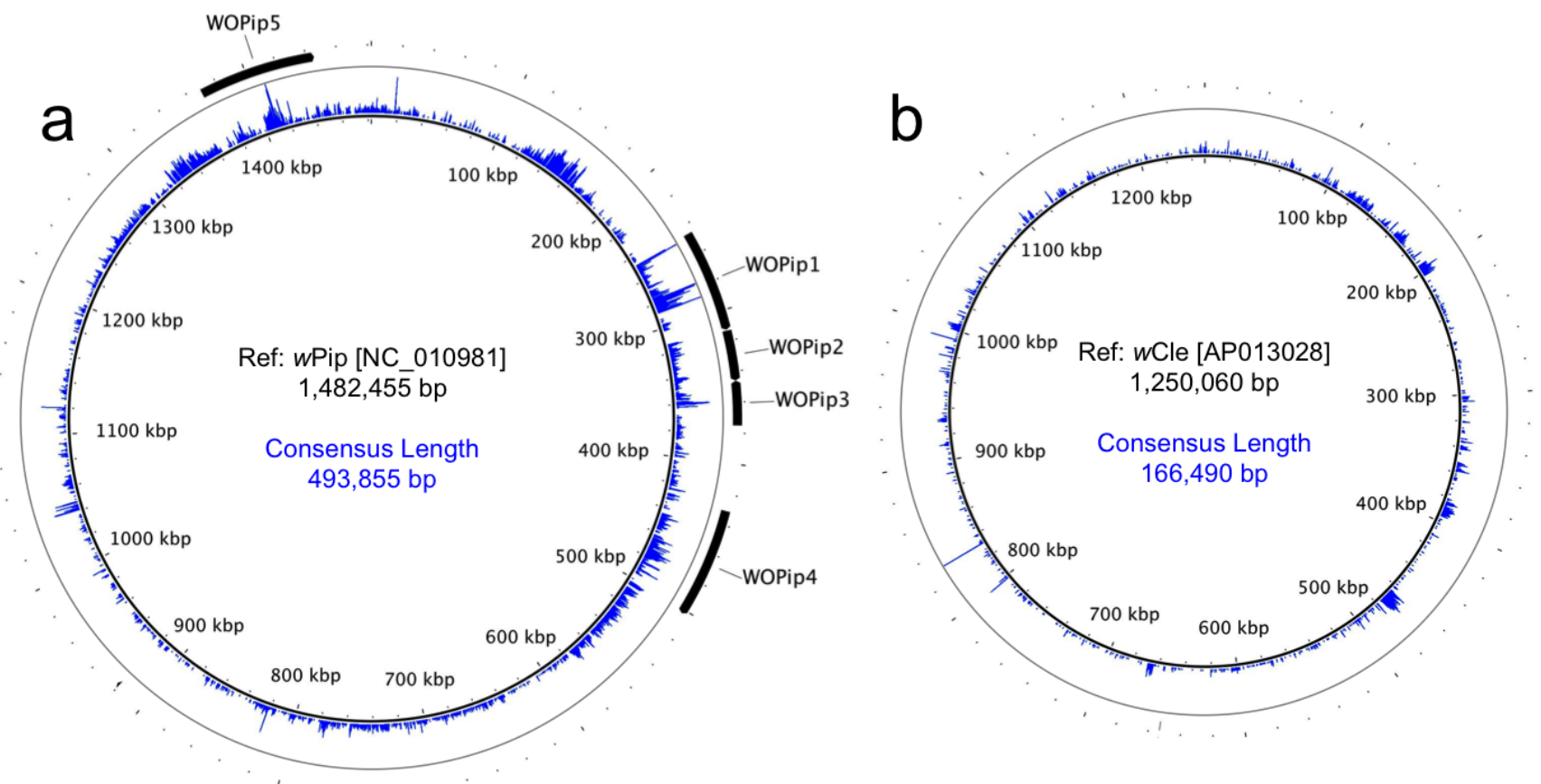

791

792 Figure 6. Circular maps of sequencing coverage across the reference genomes of (a) $w$ Pip

793 and (b) wCle. Mapping coverage at each base is represented in blue on the inner rings with the

794 max coverage set at 30 (outer gray circles). WO phage regions are indicated with black arrows. 


\section{Cpe}

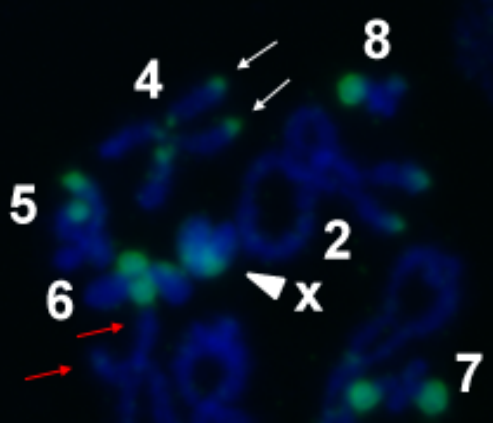

3

a

1

Figure 7. Wolbachia inserts localized to . parallelus chromosomes. Tyramide-coupled FISH

799 Fluorescein signals using the Cpar-Wb1 probe reveal presence of Wolbachia genomic inserts

800 (green fluorescence) in C. parallelus erythropus, Cpe (a) and C. parallelus parallelus, Cpp (b)

801 meiotic chromosomes (blue fluorescence). Hybridization of Wolbachia insertions is abundant in

802 telomeric regions of several chromosomes, certain interstitial regions and on chromosome $\mathrm{X}$

803 (arrowhead). White arrows mark a Wolbachia insert that coincides in homologous chromosomes

804 of both Cpe and Cpp, while red arrows indicate a subspecies-specific insert present in Cpp but

805 not Cpe. Numbers correspond to chromosome pairs (bivalents). Scale bar $=40 \mu \mathrm{m}$.

806 